\title{
SOME REMARKS ON THE EXCEPTIONAL SIMPLE LIE GROUP $\mathfrak{\Im}_{4}$
}

\author{
YOZÔ MATSUSHIMA
}

1. Let $c$ be the Cayley algebra of dimension 8 over the field $R$ of real numbers and let 3 be the set of all $3 \times 3$ Hermitian matrices

$$
X=\left(\begin{array}{lll}
\xi_{1} & x_{3} & \bar{x}_{2} \\
\bar{x}_{3} & \xi_{2} & x_{1} \\
x_{2} & \bar{x}_{1} & \xi_{3}
\end{array}\right)
$$

with coefficients in 6 . We define the multiplication in 3 by

$$
X \circ Y=\frac{1}{2}(X Y+Y X) \text {. }
$$

Then $\Im$ becomes a distributive algebra over $R$. A non-singular linear transformation $a$ of $\mathfrak{F}$ is said to be an automorphism of $\mathfrak{Y}$, if

$$
\alpha(X \circ Y)=\alpha X \circ \alpha Y
$$

for all $X, Y \in \mathcal{Y}$. The group $\mathfrak{Y}$ of all the automorphisms of $\mathcal{Y}$ is compact and the connected component containing the identity of $\mathfrak{A}$ is the exceptional simple compact group $\mathscr{F}_{40}{ }^{1 ;}$ ) Denote by $E_{i}$ the matrix (1) with $\xi_{i}=1$, all remaining terms zero. Let $\mathfrak{l}$ be the subgroup of $\hat{j}_{i}$ consisting of all automorphisms $\alpha$ such that $\alpha E_{i}=E_{i}$ for $i=1,2,3$ and let $\mathfrak{S}_{i}(i=1,2,3)$ be the subgroups of $\mathfrak{F}_{4}$ consisting of all $\alpha \in \widetilde{F}_{4}$ such that $\alpha E_{i}=E_{i}$. Then the left coset spaces $\widetilde{F}_{4} / \mathscr{S}_{i}$ are homomorphic to the set $\Pi$ of all irreducible idempotents of $\Im$ and $\Pi$ is geometrically the "plan projectif des octaves."

In this note we prove the following two theorems.

THEOREM $1 . \Re$ is connected and isomorphic to the universal covering group $\widetilde{S O}(\overline{8)}$ of the proper orthogonal group $S O(8)$ of 8 dimensional euclidean space.

ThEOREM 2. $\mathfrak{S}_{i}$ are connected and isomorphic to the universal covering group $\overparen{S O}(9)$ of the proper orthogonal group SO(9) of 9 dimensional euclidean space.

Theorem 2 gives a proof of a result anounced by A. Borel."

Received October 22, 1951.

1) See, Chevalley-Schafer [2] and Freudentha] [3].

2) See, Freudenthal [3] $\$ 7$ and Hirsch [4].

3) See, Borel [1], Théorème 1. 
2. Proof of Theorem 1. Let $F_{i}^{a}$ be the matrix (1) with $x_{i}=a$ and all numbers except $x_{i}$ zero. Then $E_{i} \circ F_{i}^{a}=0, E_{j} \circ F_{i}^{a}=\frac{1}{2} F_{i}^{a}$ if $i \neq j$. Let $\alpha \in \Re$. Then $E_{i} \circ \alpha F_{i}^{a}=0$ and $E_{j} \circ \alpha F_{i}^{a}=\frac{1}{2} \alpha F_{i}^{a}$. It follows that

$$
\alpha F_{i}^{a}=F_{i}^{\alpha_{i} a}, \quad(i=1,2,3),
$$

where $\alpha_{i}$ are the linear transformtions of $\$$.

Now $F_{i}^{a} \circ F_{i}^{b}=(a, b)\left(E_{j}+E_{k}\right){ }^{4)}$ where $\{i, j, k\}$ is a permutation of $\{1,2,3\}$, implies

$$
\left(\alpha_{i} a, \alpha_{i} b\right)=(a, b) .
$$

Denote by $O(8)$ the group of all linear transformations of $(5$ which leave the positive definite bilinear form $(a, b)$ invariant. (i.e. orthogonal transformations of (5.) Further $F_{1}^{2 x} \circ F_{2}^{2 y}=F_{3}^{2(\overline{x y})}, F_{2}^{2 x} \circ F_{3}^{2 y}=F_{1}^{2(x y)}$ and $F_{3}^{2 x} \circ F_{1}^{2 y}=F_{2}^{2(\bar{x} \bar{y})}$ imply

$$
\left\{\begin{array}{l}
\alpha_{1}(x) \alpha_{2}(y)=\kappa \alpha_{3}(x y), \\
\alpha_{2}(x) \alpha_{3}(y)=\kappa \alpha_{1}(x y), \\
\alpha_{3}(x) \alpha_{1}(y)=\kappa \alpha_{2}(x y),
\end{array}\right.
$$

where $\kappa \alpha_{i}(x)=\overline{\alpha_{i}(\bar{x})}$. Let $\gamma$ be the orthogonal transformation of 5 defined by $\gamma x=\bar{x}$ for all $x \in \mathfrak{r}$. Then $\kappa \alpha_{i}=\gamma \alpha_{i}$ and $\alpha_{i} \rightarrow \kappa \alpha_{i}$ is an automorphism of $O(8)$. We shall show that $\alpha_{i} \in S O(8)$ i.e. det. $\alpha_{i}=1$.

Lemma 1. (Frinciple of Triality. $)^{5)}$ For every $\theta \in S O(8)$, there exist $\theta_{1}$ and $\theta_{2}$ in $S O(8)$ such that

$$
\theta(x) \theta_{1}(y)=\theta_{2}(x y)
$$

for all $x, y \in\left(5\right.$. If there exist the other $\theta_{1}^{\prime}$ and $\theta_{2}^{\prime}$ in $S O(8)$ such that $\theta(x) \theta_{1}^{\prime}(y)$ $=\theta_{2}^{\prime}(x y)$, then $\theta_{1}^{\prime}= \pm \theta_{1}$ and $\theta_{2}^{\prime}= \pm \theta_{2}$. The same holds also, if we start from $\theta_{1}$ or $\theta_{2}$ instead of $\theta$.

Lemma 2. Let $\theta_{i}$ be in $O(8)$ and let

$$
\theta_{1}(x) \theta_{2}(y)=\kappa \theta_{3}(x y)
$$

for all $x, y \in\left(5\right.$. Then $\theta_{3}(x) \theta_{3}(y)=\kappa \theta_{1}(x y)$ and $\theta_{3}(x) \theta_{1}(y)=\kappa \theta_{2}(x y)$ for all $x, y \in(5$.

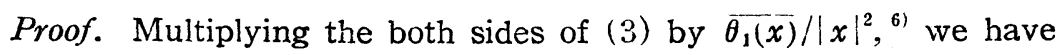

$$
\theta_{2}(y)=\frac{1}{|x|^{2}} \overline{\theta_{1}(x)} \overline{\theta_{3}(\overline{x y})} .
$$

4) The positive definite bilinear form $(a, b)$ on $\mathbb{C}$ is defined by $(a, b)=\operatorname{Re}(a b)$, where $\operatorname{Rex}=\frac{1}{2}(x+\bar{x})$.

5) See, Freudenthal [3] p. 16

6) $|x|^{2}=(x, x)=x \cdot \bar{x}=\bar{x} \cdot x$. In the following proof, we use the formulae $|\bar{x}|=|x|,|x y|$ $=|\therefore||y|, \bar{x}:(x a)=(\bar{x} x) a$ and $(a \bar{x}) x=a(\bar{x} x)$. See, Freudenthal [3] p. 7. 
Analogously we have

$$
-\frac{1}{|y|^{2}} \theta_{2}(y) \cdot \theta_{3}(\bar{y} \bar{x})=\overline{\theta_{1}(x)} \text {. }
$$

Let $\bar{x}=y z$. Then

$$
\frac{1}{|y|^{2}} \theta_{2}(y) \theta_{3}(\bar{y}(y z))=\overline{\theta_{1}(\overline{y z})} .
$$

Hence $\theta_{2}(y) \theta_{3}(z)=\kappa \theta_{3}(y z)$.

LEMma 3. Let $\theta_{i} \in O(8)(i=1,2,3)$ and $\theta_{1}(x) \theta_{2}(y)=\kappa \theta_{3}(x y)$ for all $x, y \in$ (5. Then $\theta_{i} \in S O(8)(i=1,2,3)$.

Proof. Suppose that $\theta_{1}$ is not in $S O(8)$. For every $\eta_{1} \in S O(8)$, there exist $\eta_{2}$ and $\eta_{3}$ in $S O(8)$ such that

$$
\eta_{1} \theta_{1}(x) \gamma_{2} \theta_{2}(y)=\kappa \eta_{3} \cdot \kappa \theta_{3}(x y)=\kappa\left(\eta_{3} \cdot \theta_{3}\right)(x y) .
$$

Let us choose $\eta_{1}$ such that $\eta_{1} \theta_{1}=\gamma$, where $\gamma x=\bar{x}$ for all $x \in \delta$. Then

$$
\bar{x}_{2}(y)=\kappa \zeta_{3}(x y)
$$

for all $x, y \in \mathbb{S}$, where $\zeta_{2}=\gamma_{2} \theta_{2}$ and $\zeta_{3}=\gamma_{3} \theta$. Putting $x=1$ in (4), we have $\zeta_{2}(y)$ $=\kappa \zeta_{3}(y)$. Hence $\zeta_{2}=\kappa \zeta_{3}$ and

$$
\bar{x} \zeta_{2}(y)=\zeta_{2}(x y) .
$$

Putting $y=1$ in $(5)$, we have

$$
\zeta_{2}(x)=\bar{x}_{2}(1) .
$$

Let $\zeta_{2}(1)=a$. Then $a \neq 0$. It follows from (5), and (6) that $\bar{x}(\bar{y} a)=(\bar{y} \bar{x}) a$. Hence $x(y a)=(y x) a$ for all $x, y \in(5$. It follows that $a=0$ and this is a contradiction. Hence $\theta_{1} \in S O(8)$. We may prove analogously that $\theta_{2}$ and $\theta_{3}$ are also in $S O(8)$.

Thus $\alpha_{i}(i=1,2,3)$ in (2) are in $S O(8)$. Thus if $\alpha \in \Re$, then

$$
\alpha X=\left(\begin{array}{ccc}
\xi_{1} & \alpha_{3}\left(x_{3}\right) & \kappa \alpha_{2}\left(\bar{x}_{2}\right) \\
\kappa \alpha_{3}\left(\bar{x}_{3}\right) & \xi_{2} & \alpha_{1}\left(x_{1}\right) \\
\alpha_{2}\left(x_{2}\right) & \kappa \alpha_{1}\left(\bar{x}_{1}\right) & \xi_{3}
\end{array}\right),
$$

where $X$ is the matrix (1) and $\alpha_{i}$ 's satisfy the relations (2).

Conversely let $\alpha_{1}$ be an arbitrary element in $S O(8)$ and let $\alpha_{2}$ and $\alpha_{3}$ be the elements in $S O(8)$ such that $\alpha_{1}(x) \alpha_{2}(y)=\kappa \alpha_{3}(x y)$ for all $x, y \in \mathbb{C}$ (cf. Lemma 1). Then the relations (2) hold for these $\alpha_{i}$ 's by Lemma 2. Now we define the linear transformation $\alpha\left(\alpha_{1}, \alpha_{2}, \alpha_{3}\right)$ of $\Im$ by (7). For every $\alpha_{1} \in S O(8)$ we have thus two linear transformations $\alpha\left(\alpha_{1}, \alpha_{2}, \alpha_{3}\right)$ and $\alpha\left(\alpha_{1},-\alpha_{2},-\alpha_{2}\right)$ (cf. Lemma 1). We may easily verify that these linear transformations are the automorphisms of 3 and form a closed subgroup $\mathfrak{M}$ of the group $\mathfrak{A}$ of all automorphisms of 3 . It is clear that every automorphism in $\mathfrak{M}$ leaves fixed the 
elements $E_{i}(i=1,2,3)$ and $\mathfrak{M} \supseteqq \Re$. The mapping $f_{1}\left(\alpha\left(\alpha_{1}, \alpha_{2}, \alpha_{3}\right)\right)=\alpha_{1}$ is a homomorphism of $\mathfrak{M}$ onto $S O(8)$ and the kernel of $f_{1}$ consists of $\alpha(1,1,1)$ and $\alpha(1,-1,-1)^{7)}$ Let $\mathfrak{M}_{0}$ be the connected component of $\mathfrak{M}$ containing the identity. Then $f_{1}\left(\mathfrak{M}_{0}\right)=S O(8)$. Since $f_{1}^{-1}\left(\alpha_{1}\right)=\left\{\alpha\left(\alpha_{1}, \alpha_{2}, \alpha_{3}\right), \alpha\left(\alpha_{1},-\alpha_{2},-\alpha_{3}\right)\right\}$, at least one of $\alpha\left(\alpha_{1}, \alpha_{2}, \alpha_{3}\right)$ and $\alpha\left(\alpha_{1},-\alpha_{2},-\alpha_{3}\right)$ is in $\mathfrak{M}_{0}$. We shall prove that $\mathfrak{M}=\mathfrak{M}_{0}$. Suppose, on the contrary, that $\mathfrak{M} \neq \mathfrak{M}_{0}$. Since $\mathfrak{M}_{0} \cup_{\alpha}(1,-1,-1) \mathfrak{M}_{0}$ $=\mathfrak{M}, \mathfrak{M}$ consists of two connected components and $\alpha(1,-1,-1) \bar{E} \mathfrak{M}_{0}$. Now $\alpha(-1,1,-1)$ and $\alpha(-1,-1,1)$ belong to the distinct components of $\mathfrak{M}$, for otherwise $\alpha(-1,1,-1) \alpha(-1,-1,1)=\alpha(1,-1,-1)$ is in $\mathfrak{N}_{0}$. Let, for example, $\alpha(-1,-1,1) \in \mathfrak{M}_{0}$. Let $f_{3}\left(\alpha\left(\alpha_{1}, \alpha_{2}, \alpha_{3}\right)\right)=\alpha_{3}$. Then $f_{3}$ is also a homomorphism of $\mathfrak{M}$ onto $S O(8)$ and the kernel of $f_{3}$ is $\{\alpha(1,1,1), \alpha(-1,-1,1)\}$. Hence $f_{3}$ is a local isomorphism and $f_{3}\left(\mathfrak{M}_{0}\right)=S O(8)$. By assumption the kernel of $f_{3}$ is contained in $\mathfrak{M}_{0}$ and hence $\mathfrak{M}=\mathfrak{M}_{0}$ and this is a contradiction. Hence $\mathfrak{M}=\mathfrak{M}_{0}$. Moreover we have shown that $\mathfrak{M}$ is a two sheeted covering group of $S O(8)$. Hence $\mathfrak{M}$ is isomorphic to the universal covering group $\overparen{S O}(8)$ of $S O(8)$. Since $\mathfrak{M}$ is connected, $\mathfrak{M}$ is contained in $\widetilde{\digamma}_{4}$ and each automorphism in $\mathfrak{M}$ leaves fixed the elements $E_{i}$. Hence $\mathfrak{M} \cong \mathfrak{R}$. Since we have already shown that $\mathfrak{R} \supseteqq \Re$, we have $\mathfrak{M}=\mathfrak{N}$ and this completes the proof of Theorem 1 .

3. Proof of Theorem 2. Since the subgroups $\mathscr{S}_{i}$ of $\mathfrak{F}_{1}$ are conjugate to each other in $\mathfrak{F}_{4}{ }^{8)}$ it is sufficient to consider the group $\mathscr{W}_{1}$. The derivation $\delta$ of $\Im$ such that $\delta E_{1}=0$ may be represented uniquely as the sum of two derivations

$$
\delta=\tilde{A}+\Delta,
$$

where $\Delta E_{i}=0(i=1,2,3)$ and

$$
A=\left(\begin{array}{ccc}
0 & 0 & 0 \\
0 & 0 & a \\
0 & -\bar{a} & 0
\end{array}\right), \quad a \in \mathbb{E},
$$

and $\widetilde{A} X=[A, X]=A X-X A$. Conversely for each such a metrix $A, \tilde{A}$ is a derivation of $\Im$ such that $\tilde{A} E_{1}=00^{9}$ ' Since $\Delta$ 's form the Lie algebra of the group $\Re, \operatorname{dim} .\{\Delta\}=28$ and $\operatorname{dim} .\{\tilde{A}\}=8$, where $\{\Delta\}$ and $\{\tilde{A}\}$ denote the linear spaces consisting of $\Delta$ 's and $\widetilde{A}$ 's respectively. Hence the derivations which maps $E_{1}$ to 0 form a Lie algebra of dimensions 36 and this is the Lie algebra of $\mathscr{S}_{1}$. Hence $\operatorname{dim} . \mathscr{S}=36$. Now let $I$ be the set of all irreducible idempotents of $\mathfrak{Z}^{10}{ }^{10}$ Further let $\Pi_{1}$ be the set of all $X \in \Pi$ such that $E_{1} \circ X=0$. Then an element $X \in \Im$ is in $\Pi_{1}$ if and only if

?) We denote by 1 and -1 the identity transformation and the transformation defined by $x \rightarrow-x$ respectively.

8) For, there exist $\alpha$ and $\beta$ in $\mathfrak{F}_{4}$ such that $\alpha E_{1}=E_{2}$ and $\beta E_{1}=E_{3}$. See, Freudenthal [3] p. 27. This fact is also proved in the following.

9) Chevalley-Schafer [2] and Freudenthal [3] p. 20.

10) See, Freudenthal [3] $\$ 5$. Note that the set $\Pi$ is invariant under the transformations of $\mathfrak{F}_{4}$. 


$$
X=\left(\begin{array}{ccc}
0 & 0 & 0 \\
0 & \xi_{2} & x_{1} \\
0 & \bar{x}_{1} & \xi_{3}
\end{array}\right),
$$

where $\xi_{2}=\xi_{2}^{2}+x_{1} \bar{x}_{1}, \xi_{2}+\xi_{3}=1$. Then $\xi_{3}=\xi_{3}^{2}+x_{1} \bar{x}_{1}$. Hence $1=\xi_{2}^{2}+\xi_{3}^{2}+2 x_{1} \bar{x}_{1}$. Now the bilinear form $(X, Y)=S p(X \circ Y)$ defined on $\mathcal{Y}$ is positive definite and invariant under the transformations of $\mathfrak{F}_{10}{ }^{11)}$ Let $\|X\|^{2}=(X, X)$. If $X$ is the matrix (1), then $\|X\|^{2}=\sum_{i=1}^{3} \xi_{i}+2 \sum_{i=1}^{3} x_{i} \bar{x}_{1}$. Hence if $X \in \Pi_{1}$, then $\|X\|=1$. Now let $\Im_{1}$ be the 10 dimensional linear subspace of $\Im$ consisting of the matrices of the form (8), and let $S^{9}$ be the set of all $X \in \mathfrak{F}_{1}$ such that $\|X\|=1$. Then $S^{9}$ is a 9 dimensional sphere and $\Pi_{1}$ is the intersection of $S^{9}$ and the hyper-plane $\xi_{2}+\xi_{3}=1$ in $\Im_{1}$. Hence $\Pi_{1}$ is an 8 dimensional sphere. Let $\alpha \in \mathscr{F}_{1}$. Then $\alpha\left(E_{1} \circ X\right)=E_{1} \circ \alpha X$, hence $\alpha\left(\Pi_{1}\right)=\Pi_{1}$. Thus $\alpha$ induces a transformation $R_{\alpha}$ of the sphere $\Pi_{1}$. Since $\alpha$ is an orthogonal transformation of $\mathcal{G}, R_{\alpha}$ is an isometric transformation of $\Pi_{1}$ and hence a (proper or improper) rotation. Thus $g(\alpha)=R_{\alpha}$ is a homomorphism of $\mathscr{S}_{1}$ into the group $O(9)$. Let $\mathscr{D}$ be the kernel of $g$. Since each $\alpha \in \mathscr{D}$ leaves fixed the elements $E_{i}, \alpha$ is contained in $\Re$. Hence $\alpha(\in D)$ is of the form $\alpha=\alpha\left(\alpha_{1}, \alpha_{2}, \alpha_{3}\right)$ (see $\S 1$ ) and

$$
\alpha X=\left(\begin{array}{ccc}
0 & 0 & 0 \\
0 & \xi_{2} & \alpha_{1}\left(x_{1}\right) \\
0 & \kappa \alpha_{1}\left(\bar{x}_{1}\right) & \xi_{3}
\end{array}\right)=X
$$

for all $X \in \Pi_{1}$. We see easily that $\alpha_{1}=1$ and hence $\mathfrak{D}$ is the finite group of order 2. Since $\operatorname{dim} . \mathfrak{W}_{1}=\operatorname{dim} . O(9)=36$, the component $\mathfrak{S}_{1}^{0}$ containing the identity is mapped by $g$ onto $S O(9)$. As $\mathfrak{S}_{1}^{0} \supset \mathfrak{N D}$ by Theorem $1, \mathfrak{S}_{1}^{0}$ is a two-sheeted covering group of $S O(9)$ and hence it is isomorphic to the universal covering group $\overparen{S O(9)}$ of $S O(9)$. We may easily see that if $\mathscr{S}_{1} \neq \mathscr{F}_{1}^{0}$, then the order of the group $\mathscr{H}_{1} / \mathscr{S}_{1}^{0}$ is 2 and $g\left(\mathscr{H}_{1}\right)=O(9)$. Now the mapping

$$
X \rightarrow R X=\left(\begin{array}{ccc}
0 & 0 & 0 \\
0 & \xi_{3} & x_{1} \\
0 & \bar{x}_{1} & \xi_{2}
\end{array}\right)
$$

is an improper rotation of the sphere $\Pi_{1}$. If $\mathscr{S}_{1} \neq \mathscr{S}_{1}^{0}$, there exists $\alpha \in \mathscr{F}_{1}$ such that $\alpha X=R X$ for all $X \in I_{1}$. Then $\alpha E_{1}=E_{1}, \alpha E_{2}=E_{3}$ and $\alpha E_{3}=E_{2}$. Since $g\left(\aleph_{1}^{0}\right)=S O(9)$ and $S O(9)$ is transitive on $\Pi_{1}$, there exists $\beta \in \mathscr{S}_{1}^{0}$ such that $\beta E_{2}=E_{3}$. $\beta\left(E_{1} \circ E_{3}\right)=E_{1} \circ \beta E_{3}=0, \beta\left(E_{2} \circ E_{3}\right)=E_{3} \circ \beta E_{3}=0$ and $\beta E_{3} \circ \beta E_{3}=\beta E_{3}$ imply $\beta E_{3}=E_{2}$. Then $\beta^{-1} \alpha E_{i}=E_{i}$ for $i=1,2$, 3. Thus $\beta^{-1} \alpha \in \Re \cap \mathscr{2}_{1}^{0}$. Hence $\alpha \in \mathscr{S}_{1}^{0}$ and this is a contradiction. Thus $\mathscr{H}_{1}$ is connected and isomorphic to $\widehat{S O(9)}$.

Remark. The group of all automorphisms of $\mathfrak{S}$ is not connected. For example,

\footnotetext{
נ) See, Freudenthal [3], §4.
} 


$$
X=\left(\begin{array}{lll}
\xi_{1} & x_{3} & \bar{x}_{2} \\
\bar{x}_{3} & \xi_{2} & x_{1} \\
x_{2} & \bar{x}_{1} & \xi_{3}
\end{array}\right) \rightarrow \alpha X=\left(\begin{array}{lll}
\xi_{1} & x_{2} & \bar{x}_{3} \\
\bar{x}_{2} & \xi_{3} & x_{1} \\
x_{3} & \bar{x}_{1} & \xi_{2}
\end{array}\right)
$$

is an automorphism of $3 . \alpha$ is an improper orthogonal transformation of $i$ and hence $\alpha \bar{E} \tilde{F}_{4}$.

\section{REFERENCES}

[1] Borel, A., Le plan projectif des octaves et les sphères comme espaces homogènes, C. R. Paris, (1950).

[2] Chevalley, C. and Schafer, R. D., The exceptional simple Lie algebras $F_{4}$ and $E_{6}$, Proc. Nat. Acad. Sci. U.S.A. 36 (1950).

[3] Freudenthal, H., Oktaven, Ausnahmegruppen und Oktavengeometrie, Mathematisch Instituut der Rijksuniversiteit te Utrecht, (1951).

[4] Hirsch, G., La géométrie projective et la topologie des espaces fibrés, Colloque international de topologie algebrique, Paris, (1947).

Mathematical Institute, Nagoya University 